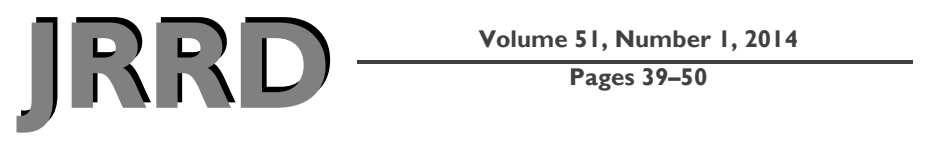

\title{
Prediction of responders for outcome measures of Locomotor Experience Applied Post Stroke trial
}

\begin{abstract}
Bruce H. K. Dobkin, MD; ${ }^{*}$ Stephen E. Nadeau, MD; ${ }^{2-3}$ Andrea L. Behrman, PT, PhD; ${ }^{4}$ Samuel S. Wu, PhD; ${ }^{5}$ Dorian K. Rose, PT, PhD; ${ }^{3,6}$ Mark Bowden, PT, PhD; ${ }^{7}$ Stephanie Studenski, PT, PhD; ${ }^{8}$ Xiaomin Lu, PhD; Pamela W. Duncan, PT, PhD $^{9}$

${ }^{1}$ Department of Neurology, Geffen School of Medicine, University of California Los Angeles, Los Angeles, CA;

${ }^{2}$ Department of Neurology, College of Medicine, University of Florida, Gainesville, FL; ${ }^{3}$ Research Service, Malcom Randall Department of Veterans Affairs (VA) Medical Center, Gainesville, FL; ${ }^{4}$ Department of Neurological Surgery, University of Louisville, Louisville, KY; ${ }^{5}$ Department of Biostatistics, University of Florida, Gainesville, FL;

${ }^{6}$ Department of Physical Therapy, University of Florida, Gainesville, FL; ${ }^{7}$ Department of Health Science and Division of Physical Therapy, Medical University of South Carolina, and Ralph H. Johnson VA Medical Center, Charleston, SC; ${ }^{8}$ Department of Internal Medicine, University of Pittsburgh, and Geriatric Research Education and Clinical Centers, VA Pittsburgh Health System, Pittsburgh, PA; ${ }^{9}$ Department of Neurology, Wake Forest University, Winston-Salem, NC
\end{abstract}

Abstract-The Locomotor Experience Applied Post Stroke rehabilitation trial found equivalent walking outcomes for body weight-supported treadmill plus overground walking practice versus home-based exercise that did not emphasize walking. From this large database, we examined several clinically important questions that provide insights into recovery of walking that may affect future trial designs. Using logistic regression analyses, we examined predictors of response based on a variety of walking speed-related outcomes and measures that captured disability, physical impairment, and quality of life. The most robust predictor was being closer at baseline to the primary outcome measure, which was the functional walking speed thresholds of $0.4 \mathrm{~m} / \mathrm{s}$ (household walking) and $0.8 \mathrm{~m} / \mathrm{s}$ (community walking). Regardless of baseline walking speed, a younger age and higher Berg Balance Scale score were relative predictors of responding, whether operationally defined by transitioning beyond each speed boundary or by a continuous change or a greater than median increase in walking speed. Of note, the cutoff values of 0.4 and $0.8 \mathrm{~m} / \mathrm{s}$ had no particular significance compared with other walking speed changes despite their general use as descriptors of functional levels of walking. No evidence was found for any difference in predictors based on treatment group.
Clinical Trial Registration: ClinicalTrials.gov; NCT00243919, "Locomotor Experience Applied Post Stroke Trial”; http://www.clinicaltrials.gov

Key words: community ambulation, exercise, functional walking level, gait speed, LEAPS, outcome measures, physical therapy, quality of life, stroke rehabilitation, walking.

Abbreviations: $\mathrm{ABC}=$ Activities-Specific Balance Confidence, ADL/IADL = activities of daily living/instrumental activities of daily living, BBS = Berg Balance Scale, E-LTP = early-LTP, F-M = Fugl-Meyer, HEP = Home Exercise Program, LEAPS = Locomotor Experience Applied Post Stroke, LL = lower limb, L-LTP = late-LTP, LTP = Locomotor Training Program, $\mathrm{mRS}=$ modified Rankin Scale, NIHSS = National Institutes of Health Stroke Scale, OR = odds ratio, RCT $=$ randomized controlled clinical trial, SIS $=$ Stroke Impact Scale, UL = upper limb.

*Address all correspondence to Bruce H. K. Dobkin, MD; Department of Neurology, Geffen School of Medicine, UCLA, 710 Westwood Plaza, Los Angeles, CA 90095; 310-206-6500; fax: 310-794-9486. Email: bdobkin@mednet.ucla.edu http://dx.doi.org/10.1682/JRRD.2013.04.0080 


\section{INTRODUCTION}

Single, randomized controlled clinical trials (RCTs) are far from the perfect instrument to determine the efficacy of a new therapeutic intervention [1]. The results of stroke rehabilitation trials can be especially challenging to interpret, partly because the level of impairment in the comparison groups, the components of the intervention, and the relevance of outcome measurement tools to the intervention bear complex interrelationships [2]. Even if a trial shows equivalence for different treatments, clinicians may ask whether a subgroup of participants was especially responsive [3]. The underlying concern is that a particular type of rehabilitation training may not have a uniform effect, so participants who fall into separable subgroups may experience differential responses to each RCT intervention. Observational practice-based evidence is another method that aims to examine the heterogeneity of responses outside of a formal trial [4]. Between-subgroup differences and within-subgroup similarities, however, are usually identifiable only by post hoc analysis, with its inherent statistical confounds [5]. Regression analyses are the most robust approach to assessing what has been called the heterogeneity effect [6]. They can capture the likelihood that patients differ from one another in multiple variables simultaneously. The establishment of baseline predictors of response to a particular therapy for walking impairment after stroke could aid clinical decisions as to whether or not to employ that therapy. It could also inform future stroke rehabilitation research.

A related question is whether the optimal outcome measurement tool was employed as the primary outcome for the RCT [7]. For a complex physical intervention, which is typical of most neurorehabilitation trials, a single measurement may not be adequate to reveal differential effects on impairment, disability, activity, and participation [8-9]. This issue can also be addressed by secondary analyses of a large database.

The Locomotor Experience Applied Post Stroke (LEAPS) RCT randomized 408 participants 2 mo after a hemiparetic stroke to two conceptually different physical therapy interventions to test for efficacy of one over the other in improving walking-related outcomes [10]. Participants were stratified to a severe impairment group if baseline speed was $<0.4 \mathrm{~m} / \mathrm{s}$ or to a moderate impairment group if speed was 0.4 to $0.8 \mathrm{~m} / \mathrm{s}$. Prior research suggested that these walking speeds are associated with home-only and limited community ambulation, respectively [11-13]. The primary outcome measurement in
LEAPS was the proportion of participants in each treatment group that transitioned to a higher functional level of walking ( $>0.4 \mathrm{~m} / \mathrm{s}$ for the severe group $>0.8 \mathrm{~m} / \mathrm{s}$ for the moderate group). The trial revealed that the two interventions were equally efficacious $[10,14]$.

In this series of secondary analyses, we addressed several clinically meaningful questions. First, we asked whether any baseline variables predicted whether a participant would transition to a higher functional walking level. Second, because a transition might not be the optimal measure of improvement, we asked whether any baseline variables predicted favorable responses as defined by tools commonly used in stroke trials, including changes in walking speed, greater than median gains in walking speed and step counts, and subscale scores on the Stroke Impact Scale (SIS) [15], which might plausibly correlate with quality of life associated with mobility. Third, we asked to what extent the transition to a higher functional walking level was associated with gains in SIS subscale scores that seem relevant to walking. Finally, we sought to determine whether the highly regarded transition boundaries chosen for the LEAPS trial $(0.4$ and $0.8 \mathrm{~m} / \mathrm{s})$ had intrinsic clinical significance in terms of their relationship to changes in SIS subscale scores or whether the significance of the boundaries lay solely in their relationship to progressive increments in walking speed. Because participants with different baseline attributes might have responded differentially to the type and timing of the LEAPS interventions, we looked for interaction effects between treatment arm and predictor variables.

\section{METHODS}

\section{Locomotor Experience Applied Post Stroke Trial}

The LEAPS trial was a multicenter, single-blind RCT that compared a Locomotor Training Program (LTP) delivered at 2 mo (early-LTP [E-LTP]) or 6 mo (late-LTP [LLTP]) poststroke in an outpatient facility with a Home Exercise Program (HEP) delivered at 2 mo at the participant's home. LTP included stepping on a treadmill with partial body weight-support for 20 to $30 \mathrm{~min}$ at $0.89 \mathrm{~m} / \mathrm{s}$ and manual assistance as needed, followed by progressive overground training for $15 \mathrm{~min}$, provided by a physical therapist and up to two rehabilitation technicians. HEP included progressive flexibility, joint range of motion, upper-limb (UL) and lower-limb (LL) strengthening, coordination, and static and dynamic balance exercises provided by a physical therapist in the home. No specific walking activity was 
undertaken in the HEP protocol. In addition to the LTP and HEP interventions, all participants could receive prescribed customary care. The LTP and HEP programs were controlled for exercise frequency (90 min sessions, 3 times per week) and duration (12 to $16 \mathrm{wk}$ ) over 30 to 36 sessions. At the time of randomization, participants had residual paresis in the LL, could walk $10 \mathrm{ft}$ with no more than one-person assistance, and had a self-selected $10 \mathrm{~m}$ walking speed of less than $0.8 \mathrm{~m} / \mathrm{s}$.

The LEAPS protocol and primary outcomes have been reported $[10,16]$. Participants were randomized $63.8 \pm 8.5 \mathrm{~d}$ poststroke; 53.4 percent walked $<0.4 \mathrm{~m} / \mathrm{s}$ and 46.6 percent walked 0.4 to $0.79 \mathrm{~m} / \mathrm{s}$. At $12 \mathrm{mo}$, 52 percent of all participants had increased functional walking ability, as defined by transitioning beyond a boundary, but no significant differences were found between the effects of E-LTP, L-LTP, and HEP. Mean walking speed at that time was approximately $0.24 \mathrm{~m} / \mathrm{s}$ higher than at baseline.

\section{Statistical Analysis}

Predictors of a transition were identified based on univariate and multivariate logistic regression analyses with backward selection of predictors. Potential interaction effects between the selected predictors and training group were examined to test whether participants with different baseline attributes responded differentially to the treatments. The dependent variable was whether a participant transitioned from one level of walking ability to a higher one. The independent variables, assessed at baseline (2 mo poststroke), included demographic measures (age, sex), training group (E-LTP, L-LTP, and HEP), side of lesion, impairment severity (moderate or severe), baseline walking speed difference from 0.4 or $0.8 \mathrm{~m} / \mathrm{s}$ for the two severity groups, National Institutes of Health Stroke Scale (NIHSS), Fugl-Meyer (F-M) LL and UL scores, Berg Balance Scale (BBS) score, modified Rankin Scale (mRS) score, and difference between the times to complete part B and part A of the Trail Making test. Need for rehospitalization and serious adverse events during the trial were included as independent variables.

We also conducted logistic regression analyses to identify predictors of response defined by a greater than median change in walking speed, SIS participation score, SIS activities of daily living/instrumental activities of daily living (ADL/IADL) score, and SIS mobility score. Furthermore, we performed a linear regression analysis, employing the same predictor variables, to identify predictors of gains in continuous walking speed. We used t-tests to compare SIS subscale scores, F-M domain scores, total steps as determined by an inertial step activity monitor, BBS, and Activities-Specific Balance Confidence (ABC) scale scores between responders and nonresponders as defined by transition beyond a boundary. Finally, to seek evidence of whether the boundary values of 0.4 and $0.8 \mathrm{~m} / \mathrm{s}$ had intrinsic ecological validity, validity that might differ as a function of baseline walking speed, we plotted several SIS measures (rating of ability to walk a block, and ADL/IADL, mobility, and participation scale scores) as a function of walking speed at 12 mo poststroke for each baseline walking speed from 0 to $0.8 \mathrm{~m} / \mathrm{s}$ in $0.1 \mathrm{~m} / \mathrm{s}$ increments. No statistical corrections were made for multiple comparisons.

\section{RESULTS}

The mean walking speed change for the 212 responders (those who achieved a transition) was $0.39 \pm 0.17 \mathrm{~m} / \mathrm{s}$ and $0.08 \pm 0.13 \mathrm{~m} / \mathrm{s}$ for nonresponders $(p<0.001)$.

\section{Predictors of Transitioning to Higher Functional Walking Level}

Table 1 shows that the smaller the difference between baseline walking speed and each of the boundary values $(0.4$ and $0.8 \mathrm{~m} / \mathrm{s}$ for severe and moderate groups, respectively), the greater the likelihood of transitioning past that boundary. This nearness-to-the-boundary benefit was found for pooled data and for each intervention. Specifically, for every increment of $0.1 \mathrm{~m} / \mathrm{s}$ between baseline walking speed and a boundary value, the odds ratio (OR) of transitioning to a higher functional level of walking decreased by 66 percent. This was the most robust predictor. Other individual predictors for transitioning were lower age ( $>50 \%$ probability if below $60 \mathrm{yr}$, Figure 1) and NIHSS score, BBS score $(>50 \%$ probability if $>40$ points, Figure 1), and higher F-M LL and UL scores and mRS score, as well as the absence of recurrent hospitalization. For every increment of $1 \mathrm{yr}$ in age, the OR for a successful transition decreased by 5 percent. For every increment of 1 point in the BBS score, the OR increased by 7 percent. For every increment of 1 point in NIHSS score (lower scores mean less impairment), the OR decreased by 9 percent. For every increment of 1 point in F-M LL score, the OR increased by 7 percent. Thus, better baseline motor function was associated with better walking outcomes. The odds of a transition for those with a mRS score $<3$ were 2.9 times those with an mRS score 
JRRD, Volume 51, Number 1, 2014

Table 1.

Predictors of response as defined by transition past walking speed boundary of all subjects.

\begin{tabular}{|c|c|c|c|c|c|c|}
\hline \multirow{2}{*}{ Variable } & \multicolumn{3}{|c|}{ Univariate Model } & \multicolumn{3}{|c|}{ Final Multivariate Model } \\
\hline & Odds Ratio & $95 \%$ CI & $p$-Value & Odds Ratio & $95 \% \mathrm{CI}$ & $p$-Value \\
\hline $\begin{array}{l}\text { Difference of Baseline Walking Speed from } 0.4 \text { or } 0.8 \\
\text { (unit }=0.1 \mathrm{~m} / \mathrm{s} \text { ) }\end{array}$ & 0.34 & $0.27-0.43$ & $<0.001$ & 0.36 & $0.28-0.46$ & $<0.001$ \\
\hline Age (yr) & 0.95 & $0.94-0.97$ & $<0.001$ & 0.95 & $0.93-0.97$ & $<0.001$ \\
\hline Berg Balance Scale Score & 1.07 & $1.05-1.09$ & $<0.001$ & 1.04 & $1.02-1.06$ & $<0.001$ \\
\hline \multicolumn{7}{|l|}{ Inpatient Hospitalization Status } \\
\hline No Hospitalization vs Hospitalization with SAE & 4.18 & $1.61-10.85$ & 0.003 & - & - & - \\
\hline Hospitalization Without vs with Specific SAEs ${ }^{*}$ & 2.12 & $0.78-5.77$ & 0.14 & - & - & - \\
\hline NIH Stroke Scale Score & 0.91 & $0.86-0.96$ & $<0.001$ & - & - & - \\
\hline Fugl-Meyer LL Score & 1.07 & $1.03-1.10$ & $<0.001$ & - & - & - \\
\hline Fugl-Meyer UL Score & 1.01 & $1.00-1.02$ & 0.004 & - & - & - \\
\hline Modified Rankin Scale Score ( $<3$ vs $\geq 3$ ) & 2.89 & $1.55-5.42$ & 0.001 & - & - & - \\
\hline \multicolumn{7}{|l|}{ Group } \\
\hline Early-LTP vs HEP & 0.95 & $0.59-1.54$ & 0.84 & - & - & - \\
\hline Late-LTP vs HEP & 1.10 & $0.68-1.77$ & 0.71 & - & - & - \\
\hline Sex (male vs female) & 1.15 & $0.78-1.70$ & 0.47 & - & - & - \\
\hline Lesion Side (left vs right) & 1.02 & $0.69-1.50$ & 0.93 & - & - & - \\
\hline Trail Making Test B - Test A (s) & 0.998 & $0.996-1.00$ & 0.11 & - & - & - \\
\hline
\end{tabular}
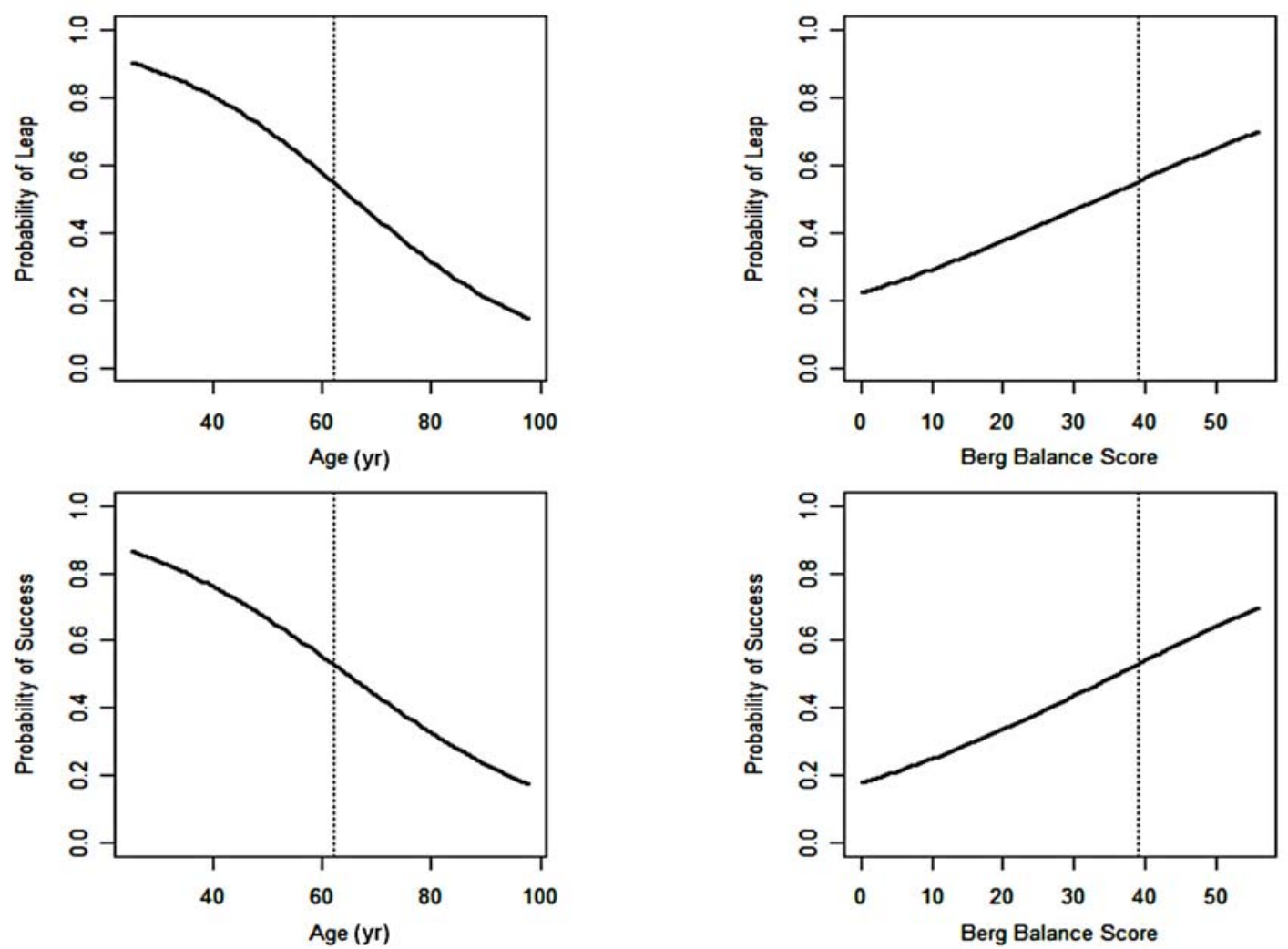

Figure 1.

Probability of transitional leap beyond 0.4 or $0.8 \mathrm{~m} / \mathrm{s}$ functional walking boundary in relation to age and Berg Balance Scale for pooled data from both interventions. Success = probability of achieving >median gain in walking speed. 
$\geq 3$ at baseline. Participants who were not hospitalized and had no serious adverse events over the course of the RCT were 4.2 times more likely to transition. However, in our multivariate logistic regression with backward selection, only the baseline-to-boundary speed difference, age, and BBS score remained significant. Finally, there were no significant interactions between these predictors and intervention group $(p>0.05)$.

\section{Predictors of Response Defined by Other Variables}

Table 2 shows the results of logistic regression analyses of predictors for responders defined by greater than a median change in walking speed, SIS participation score, SIS ADL/IADL score, or SIS mobility score. Lower age and higher BBS score were found to be significant predictors $(p<0.001)$ of a better outcome defined by greater than a median gain in walking speed. No variables predicted a greater than median change in SIS participation or ADL/IADL score. With the single exception of the relation between L-LTP and SIS mobility scale score ( $p=0.03$, uncorrected for multiple comparisons), no significant interactions between predictors and intervention group were found.

We also conducted a linear regression analysis to identify predictors of gain in continuous walking speed over the course of the trial. Age and BBS were again significant predictors, as were rehospitalization and the difference between Trail Making Tests B and A.

Of participants who achieved a greater than median gain in walking speed, 86 percent crossed a transition boundary. Of participants who achieved less than a median gain, 82 percent did not cross a transition boundary, regardless of the intervention. Thus, transitioning past a boundary and achieving a greater than median change in walking speed seemed to tap the same fundamental walking variable.

\section{Possible Clinical Meaning of Transitioning: Stroke Impact Scale Results}

Table 3 compares the SIS domains and other outcome measurements between participants who successfully transitioned to higher functional walking speeds with those who did not. Participants who did transition, regardless of initial walking level category or treatment group, had larger improvements in the SIS ADL/IADL $(p=0.01)$, hand function $(p<0.001)$, and recovery $(p<$ 0.001 ) domains, as well as higher gains in F-M UL score $(p<0.001)$, total steps walked per day $(p<0.001)$, and ABC scores $(p<0.001)$. Participants in the moderate group who transitioned also showed greater gains in SIS participation score $(p=0.048)$.

In the severe impairment group, response as defined by a greater than median gain in walking speed was also associated with greater SIS ADL/IADL and higher SIS participation scores. In the most severely impaired group (baseline speed $0-0.1 \mathrm{~m} / \mathrm{s}$ ), walking speed increases achieved by 1 yr were associated with particularly large increases in self-reported SIS mobility score.

Table 2.

Predictors of response defined as greater than median changes in walking speed and other outcomes.

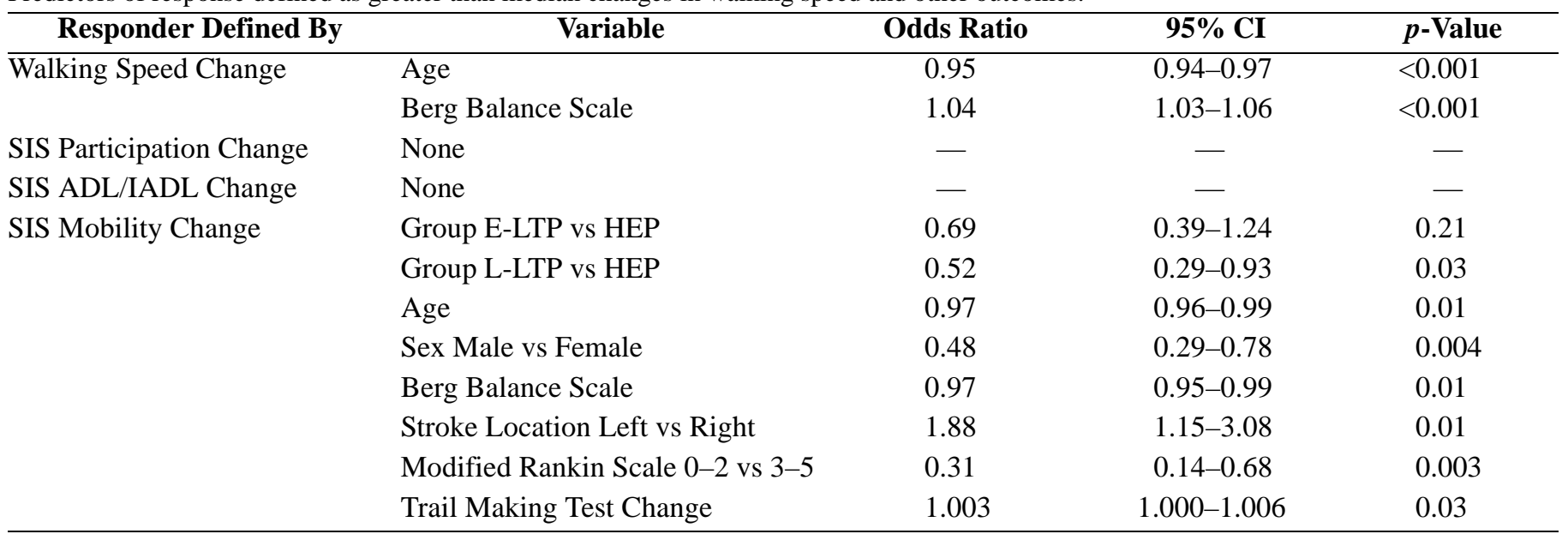


JRRD, Volume 51, Number 1, 2014

Table 3.

Comparison of Stroke Impact Scale (SIS) subscale scores and other outcomes between responders, defined by transition across walking speed boundary, and nonresponders.

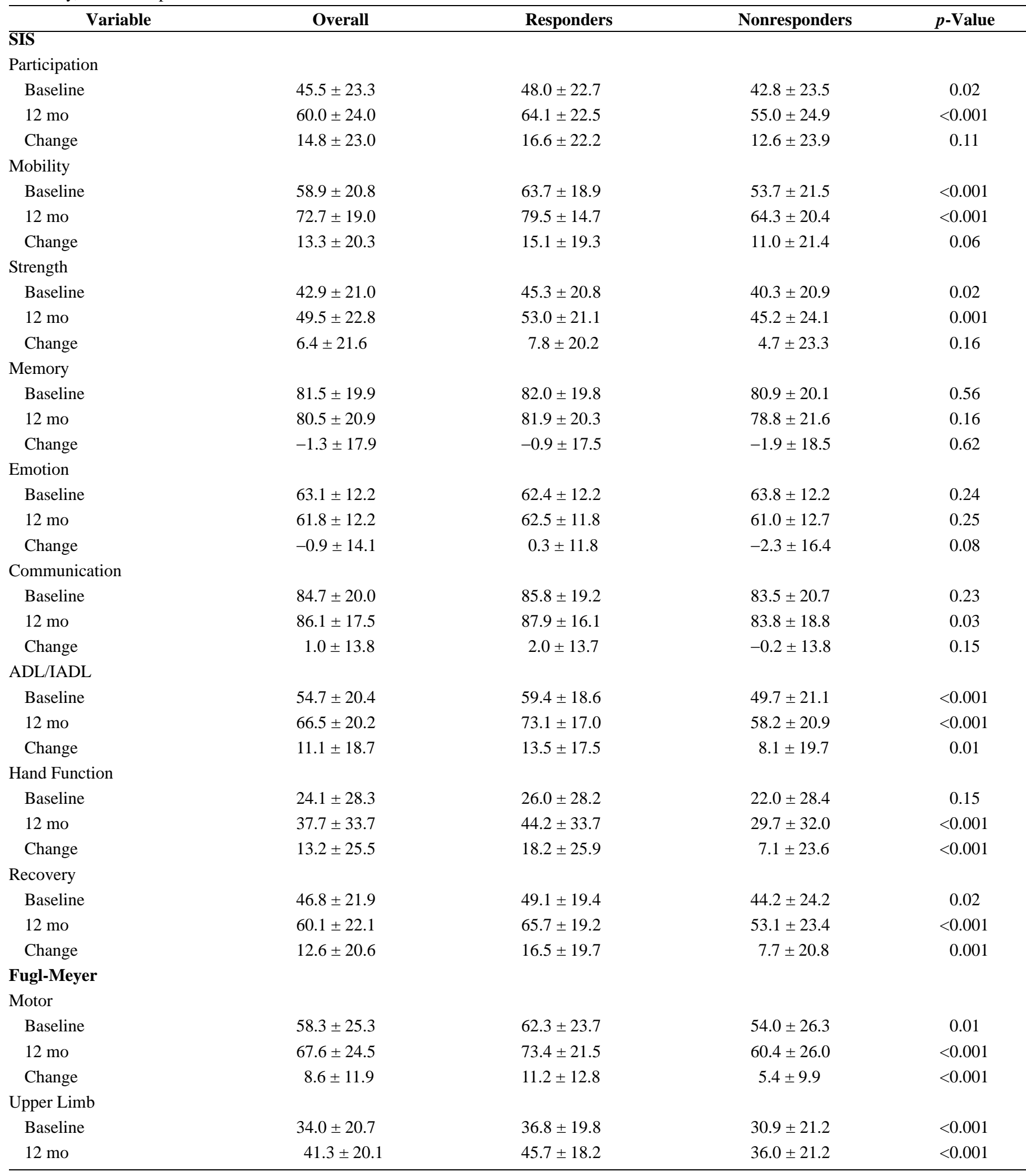


Table 3. (cont)

Comparison of Stroke Impact Scale (SIS) subscale scores and other outcomes between responders, defined by transition across walking speed boundary, and nonresponders.

\begin{tabular}{|c|c|c|c|c|}
\hline Variable & Overall & Responders & Nonresponders & p-Value \\
\hline Change & $6.8 \pm 9.9$ & $9.1 \pm 10.8$ & $3.9 \pm 8.0$ & $<0.001$ \\
\hline \multicolumn{5}{|l|}{ Lower Limb } \\
\hline Baseline & $24.4 \pm 6.4$ & $25.7 \pm 5.8$ & $23.1 \pm 6.8$ & $<0.001$ \\
\hline 12 mo & $26.3 \pm 5.8$ & $27.9 \pm 4.7$ & $24.4 \pm 6.5$ & $<0.001$ \\
\hline Change & $1.9 \pm 4.0$ & $2.2 \pm 3.9$ & $1.5 \pm 4.0$ & 0.11 \\
\hline \multicolumn{5}{|l|}{ SAM Total Steps } \\
\hline Baseline (median [Q1, Q3]) & $1,738.5[708.0,3,482.5]$ & $2,267.5[1,127.0,4,343.0]$ & $1,219.0[453.0,2,772.0]$ & \\
\hline 12 mo (mean \pm SD) & 4,294.3 $\pm 3,464.0$ & $5,403.2 \pm 3,506.1$ & $2,959.0 \pm 2,905.7$ & $<0.001$ \\
\hline 12 mo (median [Q1, Q3]) & $3,695.0[1,843.0,6,057.0]$ & $4,791.0[2,822.0,7,646.0]$ & 2,301.5 [798.0, 4,196.0] & \\
\hline Change (mean \pm SD) & $1,665.8 \pm 3,082.5$ & $2,306.6 \pm 3,337.0$ & $877.8 \pm 2,535.2$ & 0.001 \\
\hline Change (median [Q1, Q3]) & $1,097.0[-1.0,3,054.0]$ & $1,849.5[511.0,3,672.0]$ & $435.0[-265.0,1,609.0]$ & \\
\hline Change & $7.4 \pm 8.6$ & $7.9 \pm 7.7$ & $6.8 \pm 9.6$ & 0.26 \\
\hline \multicolumn{5}{|l|}{ ABC Score } \\
\hline Baseline & $45.1 \pm 23.9$ & $49.2 \pm 22.7$ & $40.6 \pm 24.3$ & $<0.001$ \\
\hline $12 \mathrm{mo}$ & $57.6 \pm 25.6$ & $66.5 \pm 21.6$ & $46.6 \pm 25.9$ & $<0.001$ \\
\hline Change & $12.3 \pm 22.0$ & $16.8 \pm 20.7$ & $6.5 \pm 22.3$ & $<0.001$ \\
\hline
\end{tabular}

\section{Ecological Validity of 0.4 and $0.8 \mathrm{~m} / \mathrm{s}$ Boundaries}

The preceding analyses suggested that transitioning past a 0.4 or $0.8 \mathrm{~m} / \mathrm{s}$ boundary did correspond to changes in walking ability that might affect quality of life. In the following analyses, we sought to determine whether the predefined transition boundaries of 0.4 and $0.8 \mathrm{~m} / \mathrm{s}$ had particular significance in their relationship to measures related to quality of life, as has been suggested in the literature. Alternatively, these boundaries might derive their significance solely from their relationship to the magnitude of gain in walking speed.

Figure 2 shows a plot of SIS item 6e (self-rated difficulty in walking a block) versus walking speed at $12 \mathrm{mo}$, stratified by baseline walking speed in bins of $0.1 \mathrm{~m} / \mathrm{s}$ at baseline. We selected this item as an alternative outcome because this self-perception may especially reflect the potential to participate in community activities. Faster walking speed was associated with less difficulty walking a block. However, visual inspection does not suggest that the cutoff values of 0.4 and $0.8 \mathrm{~m} / \mathrm{s}$ that had previously been associated with home versus community walking levels have particular significance. Rather, the ability to walk a block bears a more or less linear relationship to walking speed. This linear relationship held for all other SIS subscales tested (ADL/IADL, mobility, and participation), and in no case was there evidence of a step at 0.4 or $0.8 \mathrm{~m} / \mathrm{s}$.

Inspection of Figure 2 also reveals self-reports of walking one block being rated "not difficult at all" or "only a little difficult" despite low walking speed $(<0.4 \mathrm{~m} / \mathrm{s})$ or being rated "very difficult" despite high walking speed $(>0.8 \mathrm{~m} / \mathrm{s})$. At least 22 subjects reported the former (lowest walking speed bins, left column) and 4 reported the latter (right column).

\section{DISCUSSION}

The single strongest predictor of whether a participant had transitioned at 12 mo beyond the trial's boundaries of 0.4 and $0.8 \mathrm{~m} / \mathrm{s}$ was how close the participant had been to a boundary at baseline (2 mo after stroke). The slowest 

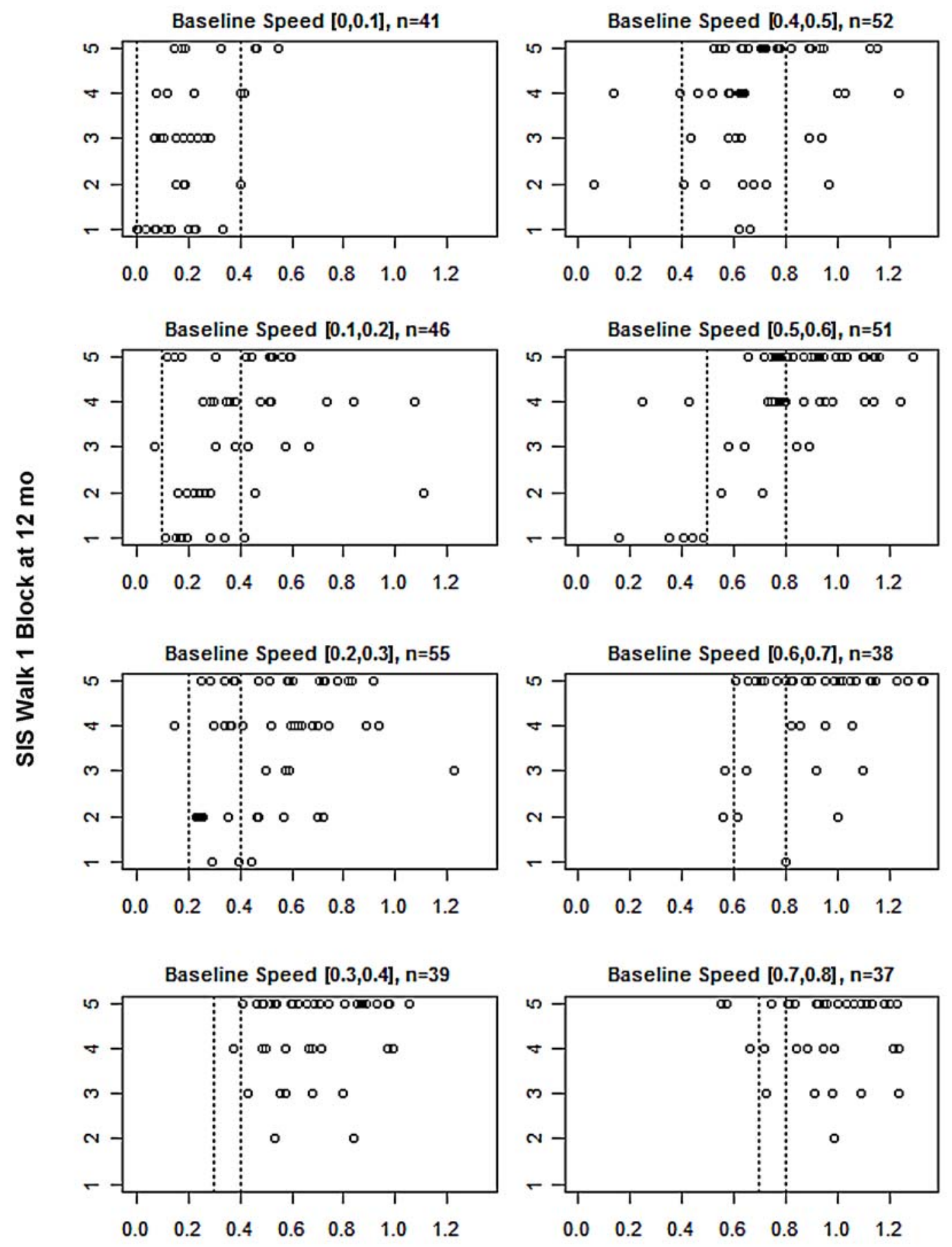

\section{Walking Speed at $12 \mathrm{mo}(\mathrm{m} / \mathrm{s})$}

\section{Figure 2.}

Plot of Stroke Impact Scale item 6e (self-rated difficulty in walking a block) by walking speed at 12 mo follow-up of all participants, stratified by baseline walking speed in bins of $0.1 \mathrm{~m} / \mathrm{s} . n=$ number of participants in that bin. Vertical broken line shows baseline speed (left) and functional walking speed boundary (right). SIS scoring: $5=$ not difficult, $4=$ a little difficult, $3=$ somewhat difficult, $2=$ very difficult, $1=$ cannot do. 
walkers in each of the two severity groups, of course, had to make greater gains on the $10 \mathrm{~m}$ walk than participants who walked faster at baseline to achieve a transition. The mean gain in walking speed across the two severity groups and the three interventions was approximately $0.24 \mathrm{~m} / \mathrm{s}$, which was not enough for the initially slowest walkers to transition past a boundary. Thus, both interventions had a ceiling effect. Another apparent limitation of both interventions was that only 15 participants, nearly all of whom walked $>0.4 \mathrm{~m} / \mathrm{s}$ at baseline, achieved a walking speed $\geq 1.2 \mathrm{~m} / \mathrm{s}$, which begins to approach the speed of nondisabled age-matched persons.

Older participants and participants who had a lower BBS score were less likely to transition past a boundary (Figure 1) regardless of treatment group. Greater age and lower BBS score were also the only consistent predictors of response defined in other ways, such as exceeding the median increase in walking speed at 12 mo or having a greater increase in walking speed. Thus, we did not identify an outcome measurement related to walking speed that would have distinguished responders from nonresponders differently than our boundary criteria for a functional transition. This finding was true for the pooled data and for each intervention.

These findings suggest several conclusions. First, although age and BBS score do not provide the basis for sharp distinctions between those who are more or less likely to respond to the types of treatment used in LEAPS, our findings do suggest that they could influence clinical decision-making. This suggestion is most clearly illustrated in Figure 1. Drawing from these data, clinicians and patients might conclude that the probability of a favorable response at advanced age and low BBS score is just too low to justify engagement in these intensive treatments. Second, more precise prediction of response will likely require measurement of additional variables that reflect yet undefined neurobiological mechanisms of recovery, along with the dynamic personal characteristics of participants as they interact with their support systems and perform in the community. Third, the potential for different baseline attributes to predict differential response to type of treatment received no support from our analyses.

\section{Stroke Impact Scale Results}

We had posited that useful baseline predictors of response might emerge more clearly if we defined response in terms of variables such as the SIS ADL/ IADL, mobility, or participation scales; self-reported ability to walk a block (SIS item 6e); or measures derived from the step activity monitor. This hypothesis was not supported. In part, this appeared to be a consequence of greater than anticipated variability in the SIS subscale reports. For example, we found 16 participants at baseline and 22 at 12 mo with walking speeds $\leq 0.4 \mathrm{~m} / \mathrm{s}$ who scored at 80 percent on SIS mobility, claiming little or no difficulty (Figure 2). Values for SIS item 6e (Figure 2), as noted, reveal instances of intuitive discrepanciesvery low speed yet little difficulty walking a block and high walking speed but much difficulty. Thus, a remarkable number of participants who started with very slow walking speeds and did not transition nonetheless reported high levels of functional mobility. The discrepancy could arise from other illness or personal issues but may represent a recalibration of response, i.e., a change in internal standards of measurement [17]. The SIS, like other health-related quality of life instruments, is also a complex tool that may allow for too much interindividual variability, despite its reported ability to detect clinically important differences [15]. Subjective and objective measures of participation have also been weakly associated in other studies [18].

\section{Functional Walking Speed Levels}

The LEAPS trial chose walking speed boundaries that represented an important change based on the results of smaller trials and observational studies [13]. These speed transitions are considered to be meaningful goals for clinical care and as trial outcomes [19]. Participants who transitioned did indeed have greater improvements across our secondary measures. While our analyses do not suggest that the threshold values of 0.4 and $0.8 \mathrm{~m} / \mathrm{s}$ have particular ecological meaning in and of themselves, they do show that transcending these thresholds bore a high correspondence to other measures of response, such as greater than median gain in walking speed. Increases in walking speed were generally associated with better self-reported quality of life, to the extent that it is reflected in the SIS ADL/IADL, mobility, and participation scores. Thus, simply walking faster confers advantages.

The LEAPS boundaries for functional walking ability derive from an early report that $0.4 \mathrm{~m} / \mathrm{s}$ serves as a threshold between household walking and the potential for community walking [12]. These findings were based on responses to SIS physical functioning types of questions, not real-world observations, at 3 mo poststroke by 147 patients who had been in stroke rehabilitation at one 
site. At 0.2 to $0.3 \mathrm{~m} / \mathrm{s}$, subjects in the study moved from limited to unlimited home walking. At 0.6 to $0.8 \mathrm{~m} / \mathrm{s}$, they moved from least limited to community-level walking. Functional ambulation self-report items, however, were in agreement with walking velocity categories only 44 percent of the time. LL strength was more discerning. The combination of walking speed and control of knee extension differentiated household from community walkers with 78 percent agreement. Thus, gait speed boundaries, alone, have perhaps been given too much weight as an outcome for clinical trials. As Perry et al. noted, the capacity for limited or full community ambulation reflects factors beyond gait speed, including the ability to negotiate uneven surfaces, curbs, and obstacles, as well as psychological and environmental interactions that may not be captured by gait speed alone, particularly on a flat laboratory walkway [12].

\section{Methodological Limitations}

Our responder versus nonresponder analysis is intrinsically limited by its post hoc nature and must be interpreted cautiously given the multiple uncorrected comparisons [6]. We also were limited to the predictor variables that we collected, although these were typical of trials that aim to improve walking after stroke.

Our primary outcome measure and the basis for this study's 0.4 and $0.8 \mathrm{~m} / \mathrm{s}$ boundaries was the $10 \mathrm{~m}$ timed walk carried out in a laboratory setting. This task, although frequently used in stroke trials, may not reflect the context and demands of walking in the home and community. Continuous monitoring of type, quantity, and quality of daily walking for practice, exercise, and travel could improve the validity of trials of walking interventions, especially by detecting all that participants do, i.e., the actual dose of formal and informal exercise and practice. Emerging techniques, employing triaxial accelerometry data analyzed by activity-recognition algorithms and managed by wireless transmission, are proving capable of remotely measuring bouts of walking or cycling speed, duration, and distance with great precision [20-23]. Thus, actual performance parameters could supplement the laboratory tasks, ordinal scales, and self-reports used in LEAPS and most rehabilitation trials.

\section{CONCLUSIONS}

Although we did not find baseline variables that sharply distinguished between those who would or would not respond to the trial interventions, lower age and higher BBS score had a sufficient influence on response to provide some basis for clinical decision-making about employing the LEAPS interventions. Closeness of individual walking speed to a transition point was the strongest predictor of transition across a boundary, but this finding reflected the fact that the further a participant was from a boundary, the greater the improvement in gait speed necessary to achieve a transition.

There was a striking lack of association between perceived walking ability and gait speed. We found no evidence of a differential relationship between baseline predictor variables and treatment group effect. The walking speed-related functional boundaries we chose appeared to be less ecologically meaningful than anticipated. More sophisticated predictor variables appear to be needed to sufficiently capture the complex relationship between the individual participant and therapeutic response. Along with seeking efficacious walking interventions for the person with more hemiparetic impairment, better measures of response are needed. The combination of quality of life and disability scales may not be sophisticated enough. Community-based measures of actual performance may more fully measure rehabilitation outcome, as well as quantify therapeutic activity by patients outside of the formal therapies being tested.

\section{ACKNOWLEDGMENTS}

\section{Author Contributions:}

Study concept and design: B. H. Dobkin, S. E. Nadeau, A. L. Behrman, S. S. Wu, D. K. Rose, P. W. Duncan.

Acquisition of data: B. H. Dobkin, S. E. Nadeau, A. L. Behrman, S. S. Wu, D. K. Rose, P. W. Duncan.

Analysis and interpretation of data: B. H. Dobkin, S. E. Nadeau, A. L. Behrman, S. S. Wu, D. K. Rose, P. W. Duncan, M. Bowden, S. Studenski.

Drafting of manuscript: B. H. Dobkin, S. E. Nadeau, A. L. Behrman, S. S. Wu, D. K. Rose, P. W. Duncan, M. Bowden, S. Studenski.

Critical revision of manuscript for important intellectual content: B. H. Dobkin, S. E. Nadeau, A. L. Behrman, S. S. Wu, D. K. Rose, P. W. Duncan, M. Bowden, S. Studenski.

Statistical analysis: S. S. Wu, X. Lu.

Obtained funding: B. H. Dobkin, S. E. Nadeau, A. L. Behrman, S. S. Wu, D. K. Rose, P. W. Duncan. 
Administrative, technical, or material support: B. H. Dobkin, S. E. Nadeau, A. L. Behrman, S. S. Wu, D. K. Rose, P. W. Duncan. Study supervision: B. H. Dobkin, S. E. Nadeau, A. L. Behrman, S. S. Wu, D. K. Rose, P. W. Duncan.

Financial Disclosures: The authors have declared that no competing interests exist.

Funding/Support: This material was based on work supported by the National Institute of Neurologic Diseases and Stroke and the National Center for Medical Rehabilitation Research (grant RO1 NS050506).

Institutional Review: The institutional review boards at all participating centers approved the protocol, and all participants provided written informed consent. An independent medical monitor and data and safety monitoring board were appointed by the National Institutes of Health.

Participant Follow-Up: The authors do not plan to inform participants of the publication of this study.

\section{REFERENCES}

1. Ioannidis JP. Contradicted and initially stronger effects in highly cited clinical research. JAMA. 2005;294(2):218-28. [PMID:16014596] http://dx.doi.org/10.1001/jama.294.2.218

2. Dobkin BH. Progressive staging of pilot studies to improve Phase III trials for motor interventions. Neurorehabil Neural Repair. 2009;23(3):197-206. [PMID:19240197] http://dx.doi.org/10.1177/1545968309331863

3. Morone G, Bragoni M, Iosa M, De Angelis D, Venturiero V, Coiro P, Pratesi L, Paolucci S. Who may benefit from robotic-assisted gait training? A randomized clinical trial in patients with subacute stroke. Neurorehabil Neural Repair. 2011;25(7):636-44. [PMID:21444654] http://dx.doi.org/10.1177/1545968311401034

4. Horn SD, Gassaway J. Practice based evidence: Incorporating clinical heterogeneity and patient-reported outcomes for comparative effectiveness research. Med Care. 2010; 48(6 Suppl):S17-22. [PMID:20421825] http://dx.doi.org/10.1097/MLR.0b013e3181d57473

5. Sun X, Briel M, Busse JW, Akl EA, You JJ, Mejza F, Bala M, Diaz-Granados N, Bassler D, Mertz D, Srinathan SK, Vandvik PO, Malaga G, Alshurafa M, Dahm P, AlonsoCoello P, Heels-Ansdell DM, Bhatnagar N, Johnston BC, Wang L, Walter SD, Altman DG, Guyatt GH. Subgroup Analysis of Trials Is Rarely Easy (SATIRE): A study protocol for a systematic review to characterize the analysis, reporting, and claim of subgroup effects in randomized trials. Trials. 2009;10:101. [PMID:19900273] http://dx.doi.org/10.1186/1745-6215-10-101

6. Kent DM, Rothwell PM, Ioannidis JP, Altman DG, Hayward RA. Assessing and reporting heterogeneity in treatment effects in clinical trials: A proposal. Trials. 2010;11:85.

[PMID:20704705]

http://dx.doi.org/10.1186/1745-6215-11-85
7. Veerbeek JM, Koolstra M, Ket JC, van Wegen EE, Kwakkel G. Effects of augmented exercise therapy on outcome of gait and gait-related activities in the first 6 months after stroke: A meta-analysis. Stroke. 2011;42(11):3311-15. [PMID:21998062] http://dx.doi.org/10.1161/STROKEAHA.111.623819

8. Mayo NE, Scott S. Evaluating a complex intervention with a single outcome may not be a good idea: An example from a randomised trial of stroke case management. Age Ageing. 2011;40(6):718-24. [PMID:22016344] http://dx.doi.org/10.1093/ageing/afr061

9. Dobkin BH. Confounders in rehabilitation trials of taskoriented training: Lessons from the designs of the EXCITE and SCILT multicenter trials. Neurorehabil Neural Repair. 2007;21(1):3-13. [PMID:17172549] http://dx.doi.org/10.1177/1545968306297329

10. Duncan PW, Sullivan KJ, Behrman AL, Azen SP, Wu SS, Nadeau SE, Dobkin BH, Rose DK, Tilson JK, Cen S, Hayden SK; LEAPS Investigative Team. Body-weightsupported treadmill rehabilitation after stroke. $\mathrm{N}$ Engl J Med. 2011;364(21):2026-36. [PMID:21612471] http://dx.doi.org/10.1056/NEJMoa1010790

11. Dickstein R. Rehabilitation of gait speed after stroke: A critical review of intervention approaches. Neurorehabil Neural Repair. 2008;22(6):649-60. [PMID:18971380] http://dx.doi.org/10.1177/15459683080220060201

12. Perry J, Garrett M, Gronley JK, Mulroy SJ. Classification of walking handicap in the stroke population. Stroke. 1995; 26(6):982-89. [PMID:7762050] http://dx.doi.org/10.1161/01.STR.26.6.982

13. Schmid A, Duncan PW, Studenski S, Lai SM, Richards L, Perera S, Wu SS. Improvements in speed-based gait classifications are meaningful. Stroke. 2007;38(7):2096-2100. [PMID:17510461] http://dx.doi.org/10.1161/STROKEAHA.106.475921

14. Dobkin BH, Duncan PW. Should body weight-supported treadmill training and robotic-assistive steppers for locomotor training trot back to the starting gate? Neurorehabil Neural Repair. 2012;26(4):308-17. [PMID:22412172] http://dx.doi.org/10.1177/1545968312439687

15. Lin KC, Fu T, Wu CY, Wang YH, Liu JS, Hsieh CJ, Lin SF. Minimal detectable change and clinically important difference of the Stroke Impact Scale in stroke patients. Neurorehabil Neural Repair. 2010;24(5):486-92. [PMID:20053950] http://dx.doi.org/10.1177/1545968309356295

16. Nadeau SE, Wu SS, Dobkin BH, Azen SP, Rose DK, Tilson JK, Cen SY, Duncan PW; LEAPS Investigative Team. Effects of task-specific and impairment-based training compared with usual care on functional walking ability after inpatient stroke rehabilitation: LEAPS Trial. Neurorehabil Neural Repair. 2013;27(4):370-80.

[PMID:23504552] http://dx.doi.org/10.1177/1545968313481284 
17. Barclay-Goddard R, Lix LM, Tate R, Weinberg L, Mayo NE. Health-related quality of life after stroke: Does response shift occur in self-perceived physical function? Arch Phys Med Rehabil. 2011;92(11):1762-69. [PMID:22032211] http://dx.doi.org/10.1016/j.apmr.2011.06.013

18. Robinson CA, Shumway-Cook A, Ciol MA, Kartin D. Participation in community walking following stroke: Subjective versus objective measures and the impact of personal factors. Phys Ther. 2011;91(12):1865-76.

[PMID:22003172]

http://dx.doi.org/10.2522/ptj.20100216

19. Management of Stroke Rehabilitation Working Group. VA/ DOD clinical practice guidelines for the management of stroke rehabilitation. J Rehabil Res Dev. 2010;47(9):1-43. [PMID:21213454]

20. Henriksen M, Lund H, Moe-Nilssen R, Bliddal H, Danneskiod-Samsøe B. Test-retest reliability of trunk accelerometric gait analysis. Gait Posture. 2004;19(3):288-97. [PMID:15125918] http://dx.doi.org/10.1016/S0966-6362(03)00069-9

21. Dobkin BH, Xu X, Batalin M, Thomas S, Kaiser W. Reliability and validity of bilateral ankle accelerometer algorithms for activity recognition and walking speed after stroke. Stroke. 2011;42(8):2246-50. [PMID:21636815] http://dx.doi.org/10.1161/STROKEAHA.110.611095
22. Patel S, Hughes R, Hester T, Stein J, Akay M, Dy J, Bonato P. Tracking motor recovery in stroke survivors undergoing rehabilitation using wearable technology. Conf Proc IEEE Eng Med Biol Soc 2010;2010:6858-61.

23. Dobkin BH, Dorsch A. The promise of mHealth: Daily activity monitoring and outcome assessments by wearable sensors. Neurorehabil Neural Repair. 2011;25(9):788-98. [PMID:21989632] http://dx.doi.org/10.1177/1545968311425908

Submitted for publication April 1, 2013. Accepted in revised form July 23, 2013.

This article and any supplementary material should be cited as follows:

Dobkin BH, Nadeau SE, Behrman AL, Wu SS, Rose DK, Bowden M, Studenski S, Lu X, Duncan PW. Prediction of responders for outcome measures of Locomotor Experience Applied Post Stroke trial. J Rehabil Res Dev. 2014;51(1):39-50.

http://dx.doi.org/10.1682/JRRD.2013.04.0080

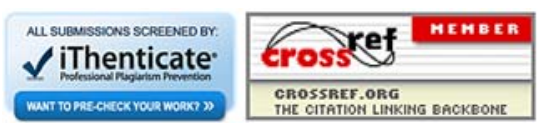

